\title{
Transmit Power Control in MIMO Cellular Systems
}

\author{
Tim W. King and Peter J. Smith \\ Department of Electrical and Computer Engineering \\ University of Canterbury, Christchurch, New Zealand \\ Email: twk20@student.canterbury.ac.nz; p.smith@elec.canterbury.ac.nz
}

\begin{abstract}
It is well known that the implementation of multiple-input multiple-output (MIMO) systems in a cellular environment is hampered by the effects of inter-cell interference. In this paper we look at controlling the base station power to increase overall system capacity. We define the optimization problem and show that the sub-optimal solution, where each base station either transmits at maximum power or is switched off, is optimal in the majority of cases. We then develop a simple and practical algorithm to find a near optimal solution. Our results show that simple power control can increase system capacity significantly, especially at high transmit power levels, and with massively reduced complexity with respect to the optimal solution.
\end{abstract}

Keywords: Optimization, Cellular Systems, MIMO, Power Control.

\section{INTRODUCTION}

In recent years, the benefits of multiple-input multiple-output (MIMO) systems have become well known. These multiple antenna links are able to provide increased performance, either in terms of throughput or quality of service, over conventional single antenna systems [1], [2], [3].

A key application of MIMO techniques is in cellular networks. However, in such systems, the intercell cochannel interference becomes a major drawback [4], [5], [6]. Recently, the use of base station (BS) collaboration has been proposed to help mitigate this interference [7], [8], [9], [10]. Possible methods include dirty paper coding [8], [11], zero-forcing beamforming [8], [9] and many others. The drawback for most of these techniques is the large amount of feedback required to convey channel state information (CSI) between the cells.

Another technique for reducing interference is transmitter power control. In the downlink this works by adjusting the total output power for each $\mathrm{BS}$, in an attempt to mitigate interference, while maintaining a satisfactory intracell link. Examples of this approach for single antenna links are given in [12], [13], [14], [15], [16]. However, as for the other techniques, optimal power control still requires BS collaboration and a significant amount of feedback and processing. Despite this, near optimal power control can exist in systems with very limited channel information at the base stations, unlike dirty paper or beamforming techniques. This leads to increased capacity without a large increase in overhead.

In this paper, we look at the problem of MIMO multicell power allocation. We begin by formulating the sum-rate equation for such a system from results in [6], [17]. Then, building on the work by Badruddin et. al. [18], we find a set of solutions to the power optimization problem. Due to the non-convexity of the aforementioned problem, no one solution is guaranteed to be globally optimal. Thus, to find the global optimum a search amongst all possible cases is needed. In order to reduce the complexity, we propose a more practical algorithm than the brute force approach. This algorithm achieves the optimal rate in the vast majority of scenarios.

Our results show that optimal power allocation is almost always the trivial case where a BS is either switched off or transmits at maximum power. Hence, the most important problem, ignoring fairness and scheduling issues, is to select the cells which should remain on. We demonstrate that this can be done extremely accurately using only the link gain information so that no CSI is required for the channel matrices. Furthermore, we show that for larger number of cells and higher SNR the scenario where one or more BSs are switched off becomes more important.

The layout of the paper is as follows. Section II gives the system model and capacity results and Sec. III formulates the optimization problem. A simplified, sub-optimal algorithm is give in Sec. IV and results are presented in Sec. V. Finally, some conclusions are given in Sec. VI.

Notation used in this paper includes $(\cdot)^{\dagger}$ for the Hermitian conjugate transpose, $\operatorname{Tr}(\cdot)$ for trace, $|\cdot|$ for determinant, $\operatorname{adj}(\cdot)$ for matrix adjugate and $(\cdot)^{*}$ for an optimal point.

\section{System Model and CAPACity Results}

In this paper we consider a set of neighbouring cells, specifically focussing on sets of 3 and 7 hexagonal cells as shown in Fig. 1. These cells each contain a single-user MIMO link with the BS located at the centre and a mobile station (MS) randomly located in the hexagon. 


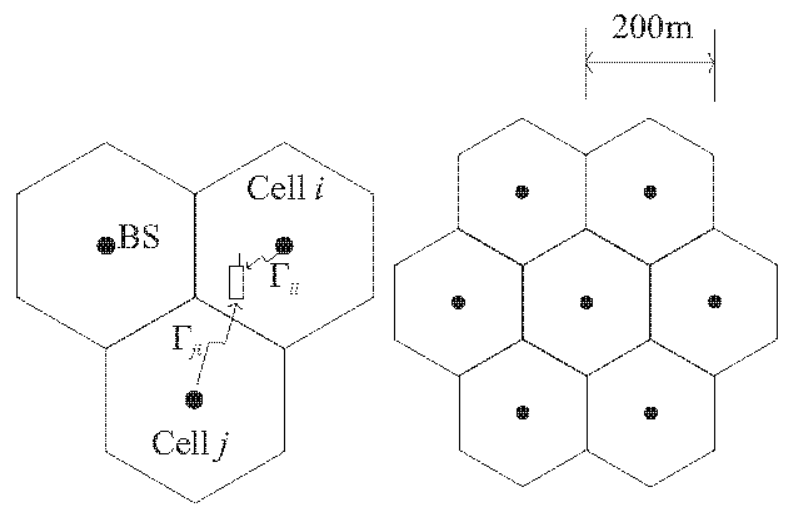

Fig. 1. Layout of both 3 cell (left) and 7 cell (right) cellular systems.

\section{A. Independent Cells}

Inside each cell we consider a single user MIMO system with $n_{t}$ transmit antennas and $n_{r}$ receive antennas. The link equation for the system is given by [1]

$$
\boldsymbol{y}=\sqrt{\Gamma} \boldsymbol{H} \boldsymbol{x}+\boldsymbol{n},
$$

where $y$ is the $n_{r} \times 1$ received signal vector, $x$ is the complex $n_{t} \times 1$ transmitted signal vector, $\boldsymbol{n}$ is an $n_{r} \times 1$ additive white complex Gaussian noise vector with magnitude variance $\sigma^{2}$ and $\boldsymbol{H}$ is a $n_{r} \times n_{t}$ complex channel matrix. In (1), $\Gamma$ is the link gain, which incorporates shadowing and path loss effects. Throughout the paper, we assume that the entries of all $\boldsymbol{H}$ matrices are zero-mean independent and identically distributed (i.i.d.) complex Gaussian variables with unit magnitude variance. Note that this assumption is for simulation purposes only and the methodology is valid for all channel models. We consider only the single user system where there is no knowledge of CSI at the transmitter. This has the capacity [1],

$$
C=\log _{2}\left|\boldsymbol{I}_{n_{r}}+\rho \boldsymbol{H} \boldsymbol{H}^{\dagger}\right|,
$$

where $\rho$ is the signal-to-noise ratio (SNR).

\section{B. Cell Clusters}

Now we consider a set of $K$ cells each with a single user MIMO system with $n_{t}$ transmit antennas and $n_{r}$ receive antennas. The link equation for a single-user MIMO link in a generic cell is given by

$$
y=\boldsymbol{H} \boldsymbol{x}+\boldsymbol{n}+\boldsymbol{i},
$$

where $i$ is a $n_{r} \times 1$ vector representing interference from all the other cells in the cluster. This multicell system has capacity, $C_{i}$, for cell $i$ where $C_{i}$ is given by $[17]$

$$
C_{i}=\log _{2} \frac{\left|\boldsymbol{I}_{n_{r}}+\rho_{i} \Gamma_{i} \boldsymbol{W}_{i}+\sum_{j=1, j \neq i}^{K} \rho_{j} \Gamma_{j i} \boldsymbol{W}_{j i}\right|}{\left|\boldsymbol{I}_{n_{r}}+\sum_{j=1, j \neq i}^{K} \rho_{j} \Gamma_{j i} \boldsymbol{W}_{j i}\right|},
$$

where $\boldsymbol{W}_{i}=\boldsymbol{H}_{i} \boldsymbol{H}_{i}^{\dagger}$ and $\boldsymbol{W}_{j i}=\boldsymbol{H}_{j i} \boldsymbol{H}_{j i}^{\dagger}$. In (4), $\boldsymbol{H}_{i}$ is the complex channel matrix for cell $i, \boldsymbol{H}_{j i}$ is the complex channel matrix between $\operatorname{BS} j$ and MS $i$, $\Gamma_{j i}$ is the link gain of the link between BS $j$ and MS $i$ and $\rho_{i}=\frac{P_{i}}{n_{+} \sigma^{2}}$ is a measure of the SNR of cell $i$ where $P_{i}$ is the transmit power of BS $i$. Note that this model assumes no CSI at the transmitter.

Whilst (4) gives the instantaneous capacity for each cell, we can calculate the mean capacity over the fast (Rayleigh) fading using [19]. Given a set of slowfading parameters [19] states that:

$\mathrm{E}_{\mathrm{H}}\left\{C_{i}\right\}=\mathcal{C}\left(K n_{t}, n_{r}, D_{1}^{(i)}\right)-\mathcal{C}\left((K-1) n_{t}, n_{r}, D_{2}^{(i)}\right)$

where $^{1}$

$$
\begin{gathered}
D_{1}^{(i)}=\operatorname{diag}\left(\rho_{1 i} \Gamma_{1 i}, \ldots, \rho_{i i} \Gamma_{i i}, \ldots, \rho_{K i} \Gamma_{K i}\right), \\
D_{2}^{(i)}=\operatorname{diag}\left(\rho_{1 i} \Gamma_{1 i}, \ldots, \rho_{K i} \Gamma_{K i}\right) .
\end{gathered}
$$

and

$$
\mathcal{C}(t, r, \Phi)=\bar{K} \sum_{k=1}^{r}\left|R^{(k)}\right| .
$$

For an explanation of the variables in (6) see Appendix A and [19]

\section{Optimization}

In any cluster of cells we wish to maximize the sum-rate whilst only adjusting the power levels at each transmitter. These levels lie within the ranges $0 \leq P_{i} \leq P_{\max , i}$, due to the transmission limitations of each BS. Without loss of generality, we assume that all the transmitters have the same maximum power, that is $P_{\max , i}=P_{\max }$. Thus, we can define the optimization problem for the sum-rate as:

$$
S^{*}=P_{i} \leq P_{\max }^{\max }, i=1, \ldots, K^{S,}
$$

where $S=\sum_{i=1}^{K} R_{i}$ and $R_{i}$ is the rate for cell $i$ which is given by [17]:

$$
R_{i}=\log _{2} \frac{\left|\boldsymbol{I}_{n_{r}}+P_{i} \bar{\Gamma}_{i} \boldsymbol{W}_{i}+\sum_{j=1, j \neq i}^{K} P_{j} \bar{\Gamma}_{j i} \boldsymbol{W}_{j i}\right|}{\left|\boldsymbol{I}_{n_{r}}+\sum_{j=1, j \neq i}^{K} P_{j} \bar{\Gamma}_{j i} \boldsymbol{W}_{j i}\right|},
$$

where $\bar{\Gamma}_{i}=\frac{\Gamma_{i}}{n_{t} \sigma^{2}}$. Defining the Lagrangian for the minimization version of (7), noting there is no sumpower constraint, gives,

$$
L(\boldsymbol{P}, \boldsymbol{\zeta})=-S-\sum_{i=1}^{K} \zeta_{1_{i}} P_{i}+\sum_{i=1}^{K} \zeta_{2_{i}}\left(P_{i}-P_{\max }\right)
$$

where $\boldsymbol{P}=\left(P_{1}, P_{2}, \ldots, P_{K}\right), \quad \zeta=$ $\left(\zeta_{1_{1}}, \ldots, \zeta_{1_{K}}, \zeta_{2_{1}}, \ldots, \zeta_{2_{K}}\right)$ and $\zeta_{(1,2)_{i}}$ are the Lagrange multipliers associated with the inequality constraints. Taking the Karush-Khan-Tucker (KKT)

${ }^{1}$ Note that the diagonal matrix, $D_{2}^{(i)}$, does not contain the direct link term, $\rho_{i \dot{i}} \Gamma_{\dot{i} i}$. 
conditions associated with (8) gives [20],

$$
\begin{aligned}
& -P_{i}^{*} \leq 0 \quad P_{i}^{*}-P_{\max }^{*} \leq 0 \quad \zeta_{1_{i}}^{*} \geq 0 \quad \zeta_{2_{i}}^{*} \geq 0 \\
& \zeta_{1_{i}}^{*} P_{i}^{*}=0 \quad \zeta_{2_{i}}^{*}\left(P_{i}^{*}-P_{\max }^{*}\right)=0 \\
& \frac{\partial S^{*}}{\partial P_{i}^{*}}-\zeta_{1_{i}}^{*}+\zeta_{2_{i}}^{*}=0 .
\end{aligned}
$$

Taking the final KKT condition in (10) and multiplying by $P_{i}^{*}\left(P_{i}^{*}-P_{\max }^{*}\right)$ gives:

$$
\begin{aligned}
P_{i}^{*}\left(P_{i}^{*}-P_{\max }^{*}\right) \frac{\partial S^{*}}{\partial P_{i}^{*}} & -P_{i}^{*}\left(P_{i}^{*}-P_{\max }^{*}\right) \zeta_{1_{i}}^{*} \\
& +P_{i}^{*}\left(P_{i}^{*}-P_{\max }^{*}\right) \zeta_{2_{i}}^{*}=0
\end{aligned}
$$

Using the $5^{\text {th }}$ and $6^{\text {th }} \mathrm{KKT}$ conditions in (10), equation (11) gives

$$
P_{i}^{*}\left(P_{i}^{*}-P_{\max }^{*}\right) \frac{\partial S^{*}}{\partial P_{i}^{*}}=0 .
$$

Equation (12) has three sets of solutions:

$$
P_{i}^{*}=0 \quad P_{i}^{*}=P_{\max } \quad \frac{\partial S^{*}}{\partial P_{i}^{*}}=0 .
$$

Whilst the first two solutions given in (13) are straightforward, the third solution is harder to find. Setting $\boldsymbol{A}_{j i}=\bar{\Gamma}_{j i} \boldsymbol{W}_{j i}$ we can rewrite $S$ as

$$
\begin{aligned}
S= & \sum_{i=1}^{K} \log _{2}\left|\boldsymbol{I}_{n_{r}}+P_{i} \boldsymbol{A}_{i i}+\sum_{j=1, j \neq i}^{K} P_{j} \boldsymbol{A}_{j i}\right| \\
& -\sum_{i=1}^{K} \log _{2}\left|\boldsymbol{I}_{n_{r}}+\sum_{j=1, j \neq i}^{K} P_{j} \boldsymbol{A}_{j i}\right| .
\end{aligned}
$$

Using the property, $\frac{\partial}{\partial P} \log |P \boldsymbol{X}+\boldsymbol{Y}|=\operatorname{Tr}((P \boldsymbol{X}+$ $\boldsymbol{Y})^{-1} \boldsymbol{X}$ ) [21], we can differentiate (14) to give (15). Setting $\frac{\partial S^{*}}{\partial P^{*}}=0$ and solving (15) gives a polynomial of order $K^{2} n_{r}-1$ in $P_{i}$. This can be seen by writing the inverse matrices in $(15)$ as $\left(P_{i} \boldsymbol{F}+\boldsymbol{G}\right)^{-1}=$ $\operatorname{adj}\left(P_{i} \boldsymbol{F}+\boldsymbol{G}\right)\left|P_{i} \boldsymbol{F}+\boldsymbol{G}\right|^{-1}$. Multiplying through by the determinants gives a polynomial expression in $P_{i}$ which can be identified as having order $K n_{r}-1$. Since $P_{i}=0$ and $P_{i}=P_{\max }$ are also candidate solutions, there are up to $K n_{r}+1$ possible feasible values for $P_{i}$ and $K$ powers to be allocated. Furthermore, since (7) is non-convex, it is difficult to find the global maximum value without evaluation of all these possible combinations of solutions.

There are 2 fundamental problems here. Firstly, the overhead in computing all the solutions and secondly the need for full network CSI (all $\boldsymbol{W}_{i}$ and $\boldsymbol{W}_{j i}$ matrices) to compute (14) or (15). Hence we consider two trivial simplifications:

- consider only the $2^{K}$ power allocations where $P_{i} \in\left\{0, P_{\max }\right\}$;

- maximize $E_{H}(S)$ rather than $S$, so that the $\boldsymbol{W}_{i}$, $\boldsymbol{W}_{j i}$ matrices are not required.

Furthermore, to avoid the search over the $2^{K} \mathrm{ON} / \mathrm{OFF}$ possibilities we also derive a simplified algorithm

\begin{tabular}{|c|c|c|c|c|}
\hline Cell-Edge SNR $(\mathrm{dB})$ & -10 & 0 & 10 & 20 \\
\hline Percentage & 100 & 100 & 99.99 & 99.99 \\
\hline
\end{tabular}

TABLE I

PERCENTAGE OF THE OPTIMAL CAPACITY ACHIEVED BY A 2 CELL ON/OFF SELECTION SYSTEM

below.

\section{A Practical Algorithm}

To reduce the optimal search across the $2^{K}$ possible solutions $\mathrm{ON} / \mathrm{OFF}$ we propose a simple, practical algorithm for efficiently finding a near optimal sumrate. Note that the algorithm is ad-hoc in nature but not only approaches the best $\mathrm{ON} / \mathrm{OFF}$ solution but is also very close to the global optimum where results are available. The idea is to start with all BSs transmitting at $P_{\max }$. Then, one at a time, an individual BS is switched off. If all the sum-rates with $K-1$ BSs operational are the less than the original, then the policy is to use all BSs. If some of the sum-rates are higher than the original then the highest is chosen and the policy is to switch one BS off. Then the procedure is replicated to see if it beneficial to switch off another BS. This algorithm is repeated until no further gains are achieved. The approach is summarized in Algorithm 1 below. This simplified search avoids the tree structure of the optimal method and simplifies the problem from $O\left(2^{K}\right)$ capacity calculations to $O\left(K^{2}\right)$.

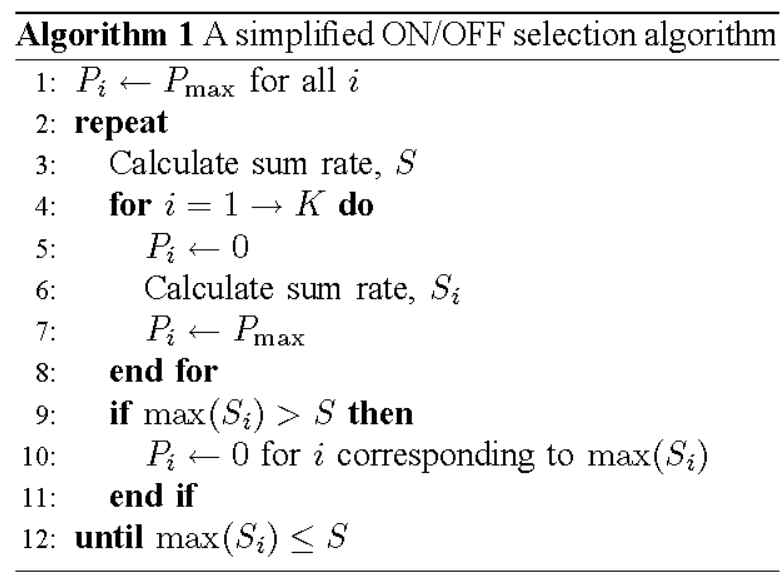

\section{RESUlts}

All simulations were carried out in a shadow fading environment with log-normal shadowing (with standard deviation $9 \mathrm{~dB}$ ) and path loss effects (with path loss exponent $\gamma=3$ ). We use the classic fading model:

$$
\Gamma_{i}=A L r^{-\gamma},
$$

where $A$ is a constant, $L$ is a lognormal shadowing variable and $r$ is the link distance. We assume a uniform user distribution in a $100 \mathrm{~m}$ internal radius hexagonal cell with a $10 \mathrm{~m}$ exclusion zone similar to that in [22]. The value, $A$, is adjusted to ensure that the mean SNR of the user at the cell edge $(r=100 \mathrm{~m})$ is 


$$
\begin{aligned}
\frac{\partial S}{\partial P_{i}}= & \frac{1}{\log 2}\left(\frac{\partial}{\partial P_{i}} \log _{2}\left|\boldsymbol{I}_{n_{r}}+P_{i} \boldsymbol{A}_{i i}+\sum_{j=1, j \neq i}^{K} P_{j} \boldsymbol{A}_{j i}\right|-\sum_{j=1, j \neq i}^{K} \frac{\partial}{\partial P_{i}} \log _{2}\left|\boldsymbol{I}_{n_{r}}+P_{i} \boldsymbol{A}_{i j}+\sum_{k \neq j, k=1}^{K} P_{k} \boldsymbol{A}_{k j}\right|\right) \\
= & \frac{1}{\log 2} \operatorname{Tr}\left(\left(\boldsymbol{I}_{n_{r}}+P_{i} \boldsymbol{A}_{i i}+\sum_{j=1, j \neq i}^{K} P_{j} \boldsymbol{A}_{j i}\right)^{-1} \boldsymbol{A}_{i i}\right) \\
& -\frac{1}{\log 2} \sum_{j=1, j \neq i}^{K} \operatorname{Tr}\left(\left(\boldsymbol{I}_{n_{r}}+P_{i} \boldsymbol{A}_{i j}+\sum_{k \neq j, k=1}^{K} P_{k} \boldsymbol{A}_{k j}\right)^{-1} \boldsymbol{A}_{i j}\right)
\end{aligned}
$$

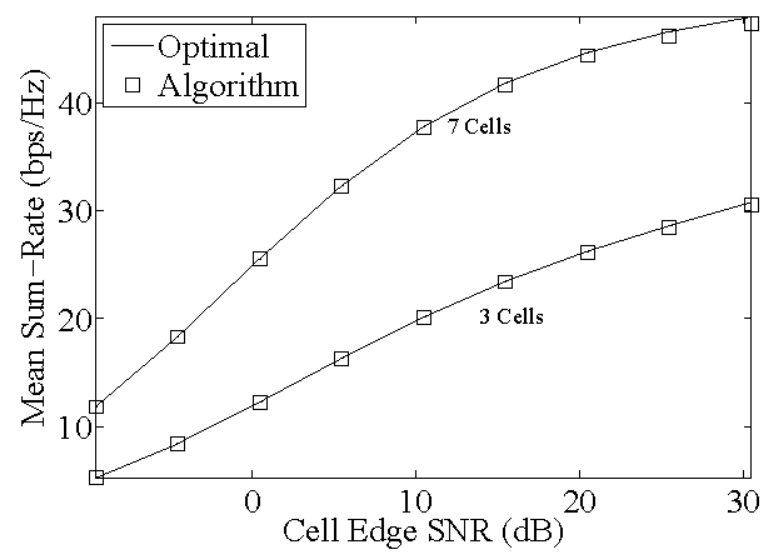

Fig. 2. A comparison of mean sum-rates for algorithm 1 with the optimal ON/OFF solution.

given by a fixed cell-edge SNR value (see Figs. 2-5). This mean SNR is averaged over the shadow fading. In each cell a $(2,2)$ MIMO system is considered so that $n_{r}=n_{t}=2$. In future work, larger MIMO systems should be considered to see whether any fundamental change in the nature of the results occurs with larger numbers of antennas.

The first set of results are shown in Table I. Here we consider a 2 cell system and find the globally optimal power allocation. This is achieved by setting $P_{1}=$ $P_{\max }$ and solving (12) for $P_{2}$ and then comparing this solution with that obtained by setting $P_{2}=P_{\max }$ and solving (12) for $P_{1}$. The largest sum-rate is the optimal power allocation since at least one of the cells must use maximum power (see Appendix B for a proof). The resulting optimal power allocation gives a sumrate which is almost identical to the sum-rate given by the simple policy where each BS is either OFF or operating at $P_{\max }$ as shown in Table I. This motivates the focus of the paper on power allocation policies which are either ON or OFF. In future work it is desirable to see if this property still holds for larger number of cells.

In Fig. 2 we compare the best $\mathrm{ON} / \mathrm{OFF}$ allocation over all $2^{K}$ possibilities with the results of Algorithm 1. In these results the rates are computed from (8) so the instantaneous channel matrices are used. For both the 3 and 7 cell scenarios the simplified algorithm is virtually optimal.

In Fig. 3 we consider 3 allocation policies. The simplest approach is "All On" where $P_{i}=P_{\max }$, for all $i=1,2, \ldots, K$. The best ON/OFF solution is labeled "Opt(Sim)" and this simulates the best sum-rate achieved by searching all $2^{K}$ possibilities using the instantaneous channel matrices. A similar policy computes $\mathrm{E}_{\mathrm{H}}(S)$ for all $2^{K}$ possibilities and records the best option. The instantaneous sum-rate for this option is then recorded. Note that this policy, denoted "Opt(Ana)", uses the analytical mean values of (8) using the result in (5). Hence, no channel matrices are required. Figure 3 shows two key points. Firstly, it shows that selection based on mean sumrates (requiring only the $\Gamma$ values), is approximately equal to the instantaneous capacity selection. This indicates that the link gains and slow fading have a more significant effect on the capacity than the fast Rayleigh fading. Secondly, it shows that power management does provide significant gains over an "All On" approach especially at higher transmit powers and for larger number of cells. Also note that as expected, the mean sum-rate for the "All On" approach reaches a ceiling when the gains due to increased power are balanced by increasing interference. Also shown in Fig. 3 are mean sum-rate values (shown by the circles) evaluated using (5). These match the simulated means for the "All On" case and provide a check on the analytical results used to select BSs in the absence of any CSI for the channel matrices.

Figure 4 shows the distribution of the number of active cells in the optimum $\mathrm{ON} / \mathrm{OFF}$ solution versus SNR for a 3 cell system. At lower SNR, when interference less of a factor, using all 3 cells simultaneously is preferred in most cases (approximately $70 \%$ of the time the "All On" approach is best at $-10 \mathrm{~dB}$ SNR). However, when the SNR increases, the use of all 3 cells decreases and the use of 2 out of 3 cells starts to dominate.

As shown above, with the optimal or sub-optimal power allocations some cells will not be active at certain points in time. To address this, a multipleaccess scheme such as TDMA can be used to give weighted time/frequency allocations to two sets of 


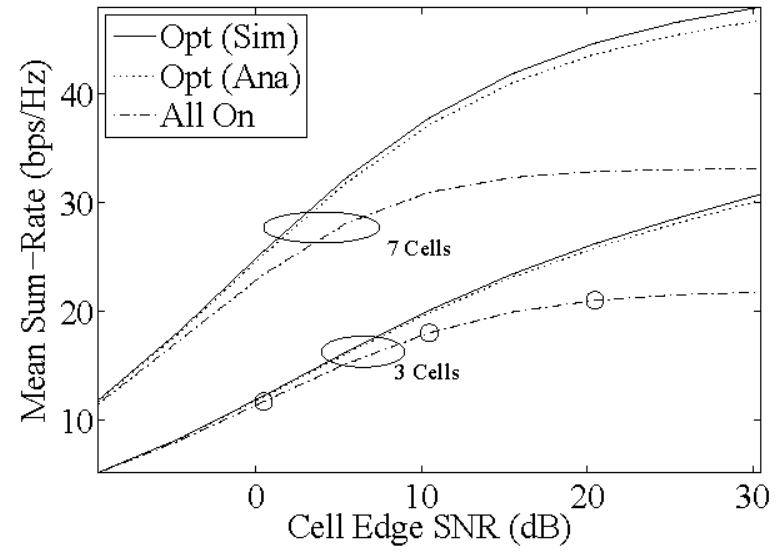

Fig. 3. A comparison of mean sum-rates for a 3 cell and 7 cell system with various allocation policies.

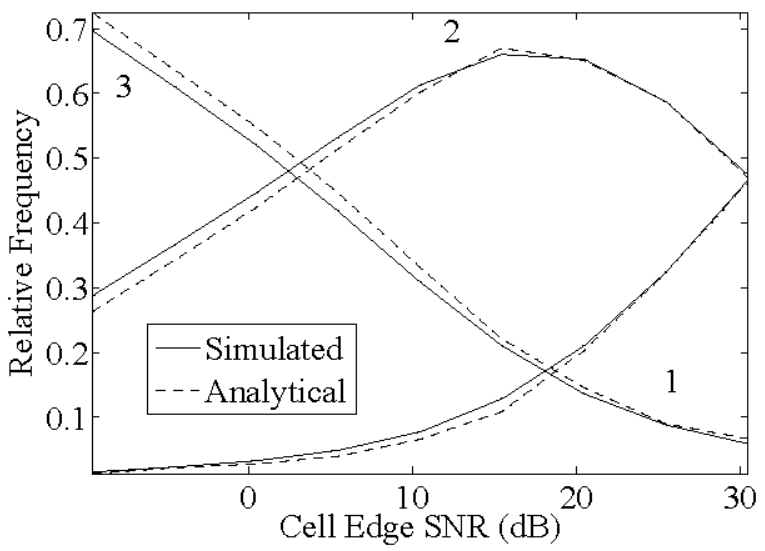

Fig. 4. Distribution of active cells for a 3 cell system (the number of active cells is labeled).

cells. Set 1 is operational in time slot 1 and set 2 is operational in time slot 2 . We consider a very simple approach where the set 1 cells operate for a proportion of time given by a reuse-factor $(\mathrm{RF})$. The set 2 cells are then operational for a proportion of time given by $1-R F$. The overall sum-rate is the weighted combination of the individual sum-rates for

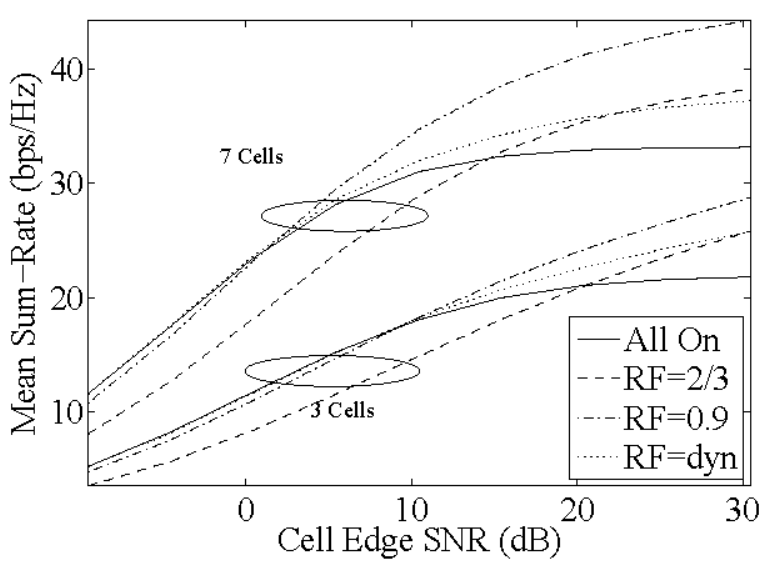

Fig. 5. A comparison of different TDMA reuse factors. the two sets of cells. The final selection of which cells are in each set is determined by choosing the maximum of the weighted sum-rates. Figure 5 shows such a TDMA scheme with various values of RF. The dynamic allocation strategy (dyn) shown in Fig. 5 uses a dynamic reuse factor equal to the number of cells in set 1 divided by the total number of cells. Figure 5 shows that sum-rate improvements can be achieved, even when fairness issues are considered. However, with the simple TDMA scheme shown, the advantages of power control are only realized at high SNR. Note that the advantages occur at lower SNR for higher numbers of cells. Hence, with coordination over a larger cluster, say 19 cells, the TDMA scheme may be beneficial at realistic cell-edge SNR values.

\section{CONCLUSion}

We have provided analysis and results for cellular MIMO power control. We have also developed a fast, near-optimal algorithm to implement power control. The results show that power control can significantly boost the system throughput, especially at higher SNRs. However, both optimal and sub-optimal power allocations often involve switching off one or more BSs. In response to this observation we have also shown that a multiple access policy such as TDMA could be used to improve system capacity whilst still providing some quality-of-service to all cells.

\section{APPENDIX A \\ VARIABLES IN THE MEAN CAPACITY EQUATION}

In (6) $\Phi$ is the transmit covariance matrix and the normalization constant $\bar{K}$ is given by [19]:

$$
\bar{K}=\frac{(-1)^{r(t-r)} \prod_{i=1}^{L} \mu_{(i)}^{m_{i} r}}{\Gamma_{(r)}(r) \prod_{i<j}\left(\mu_{(i)}-\mu_{(j)}\right)^{m_{i} m_{j}} \prod_{i=1}^{L} \Gamma_{\left(m_{i}\right)}\left(m_{i}\right)},
$$

where $\mu_{(1)}>\mu_{(2)}>\cdots>\mu_{(L)}$ are the $L$ distinct eigenvalues of $\Phi^{-1}$, with associated multiplicities $m_{1}, \ldots, m_{L}$ such that $\sum_{i=1}^{L} m_{i}=t$, and $\Gamma_{(m)}(a)=$ $\prod_{i=1}^{m}(a-i)$ !. The $t \times t$ matrix $\boldsymbol{R}^{(k)}$ has elements:

$$
\begin{aligned}
& r_{i, j}^{(k)}= \\
& \left\{\begin{array}{cc}
(-1)^{d(i)} \frac{(j+d(i)-1) !}{\mu_{(e)(i))}^{j+d(i)}} & j=1, \ldots, r ; j \neq k \\
\frac{(-1)^{d(i)}}{\log 2} \sum_{s=0}^{j+d(i)-1} q(i, j, s) & j=1, \ldots, r ; j=k \\
{[t-d(i)]_{d(i)} \mu_{(e(i))}^{t-j-d(i)}} & j=r+1, \ldots, t
\end{array},\right.
\end{aligned}
$$

where $[a]_{n} \triangleq a(a-1) \ldots(a-n+1)$. Note that $[a]_{0} \triangleq$ 1 . The function $q(i, j, s)$ is given by

$$
\begin{aligned}
q(i, j, s)= & \exp \left(\mu_{(e(i))}\right) \mathrm{E}_{1}\left(\mu_{(e(i))}\right) \\
& +\sum_{p=1}^{j+d(i)-s-1} \frac{(-1)^{j+d(i)-s-p-1}(p-1) !}{\mu_{(e(i))}^{p}}
\end{aligned}
$$


where $\mathrm{E}_{1}(x)=\int_{x}^{\infty} \frac{e^{-t}}{t} d t$ is the exponential integral. $e(i)$ is an indicator function which is defined as the unique integer such that

$$
m_{1}+\cdots+m_{e(i)-1}<i \leq m_{1}+\cdots+m_{e(i)},
$$

and

$$
d(i)=\sum_{k=1}^{e(i)} m_{k}-i .
$$

\section{Power Allocation to one Dominant UsER}

Given any cluster of cells, we show that the maximum sum-rate occurs when at least one cell has full power. For any cell, $i$, the effect of a small global increase in power can be described by, $P_{i}=(1+\varepsilon) P_{i}$. This results in a rate for cell $i, \bar{R}_{i}$, given by:

$$
\begin{aligned}
\bar{R}_{i}=\log _{2} \mid \boldsymbol{I}_{n_{r}}+(1+\varepsilon) P_{i} \bar{\Gamma}_{i} \boldsymbol{W}_{i} \\
+\sum_{j=1, j \neq i}^{K}(1+\varepsilon) P_{j} \bar{\Gamma}_{j i} \boldsymbol{W}_{j i} \mid \\
\quad-\log _{2}\left|\boldsymbol{I}_{n_{r}}+\sum_{j=1, j \neq i}^{K}(1+\varepsilon) P_{j} \bar{\Gamma}_{j i} \boldsymbol{W}_{j i}\right| .
\end{aligned}
$$

Setting $\boldsymbol{F}=P_{i} \bar{\Gamma}_{i} \boldsymbol{W}_{i}$ and $\boldsymbol{G}=\sum_{j=1, j \neq i}^{K} P_{j} \bar{\Gamma}_{j i} \boldsymbol{W}_{j i}$, we show that $\bar{R}_{i} \geq R_{i}$ or:

$\log _{2} \frac{\left|\boldsymbol{I}_{n_{r}}+(1+\varepsilon)(\boldsymbol{F}+\boldsymbol{G})\right|}{\left|\boldsymbol{I}_{n_{r}}+(1+\varepsilon) \boldsymbol{G}\right|} \geq \log _{2} \frac{\left|\boldsymbol{I}_{n_{r}}+\boldsymbol{F}+\boldsymbol{G}\right|}{\left|\boldsymbol{I}_{n_{r}}+\boldsymbol{G}\right|}$.

Rearranging (21) gives

$$
\begin{array}{r}
\left|\boldsymbol{I}_{n_{r}}+(1+\varepsilon)(\boldsymbol{F}+\boldsymbol{G})\right|\left|\boldsymbol{I}_{n_{r}}+\boldsymbol{G}\right| \geq \\
\quad\left|\boldsymbol{I}_{n_{r}}+\boldsymbol{F}+\boldsymbol{G}\right|\left|\boldsymbol{I}_{n_{r}}+(1+\varepsilon) \boldsymbol{G}\right| .
\end{array}
$$

Using the determinant property, $|\boldsymbol{A} \boldsymbol{B}|=|\boldsymbol{A}||\boldsymbol{B}|$, gives

$$
\begin{array}{r}
\left|\boldsymbol{I}_{n_{r}}+\boldsymbol{G}+(1+\varepsilon)(\boldsymbol{F}+\boldsymbol{G})+(1+\varepsilon)(\boldsymbol{F}+\boldsymbol{G}) \boldsymbol{G}\right| \geq \\
\left|\boldsymbol{I}_{n_{r}}+\boldsymbol{F}+\boldsymbol{G}+(1+\varepsilon) \boldsymbol{G}+(1+\varepsilon)(\boldsymbol{F}+\boldsymbol{G}) \boldsymbol{G}\right|,
\end{array}
$$

which reduces to

$$
|\boldsymbol{E}+\varepsilon(\boldsymbol{F}+\boldsymbol{G})| \geq|\boldsymbol{E}+\varepsilon \boldsymbol{G}|,
$$

where $\boldsymbol{E}=\boldsymbol{I}_{n_{r}}+\boldsymbol{F}+2 \boldsymbol{G}+(1+\varepsilon)(\boldsymbol{F}+\boldsymbol{G}) \boldsymbol{G}$. Since $\boldsymbol{F}$ and $\boldsymbol{G}$ are positive semi-definite, (24) holds and therefore (21) holds. This shows that a small global increase in power will increase each cell's rate and thus the sum-rate. This means that the system should increase power so that at least one is capped at $P_{\max }$.

\section{REFERENCES}

[1] I. E. Telatar, "Capacity of multi-antenna Gaussian channels," European Trans. on Telecomm. Related Technol, vol. 10, pp. 585-595, Nov.-Dec. 1999

[2] G. J. Foschini and M. J. Gans, "On limits of wireless communication in a fading environment when using multiple antennas," Wireless Personal Commun., vol. 6, no. 3, pp. 311-335, Mar. 1998.
[3] J. H. Winters, "On the capacity of radio communication systems with diversity in a Rayleigh fading environment," IEEE J. Select. Areas Commun., vol. 5, no. 5, pp. 871-878, June 1987

[4] S. Catreux, P. Driessen, and L. Greenstein, "Attainable throughput of an interference-limited multiple-input multipleoutput (MIMO) cellular system," IEEE Trans. Commun., vol. 49, no. 8, pp. 1307-1311, Aug. 2001.

[5] R. S. Blum, J. H. Winters, and N. R. Sollenberger, "On the capacity of cellular systems with MIMO," IEEE Commun. Lett., vol. 6, no. 6, pp. 242-244, June 2002.

[6] R. S. Blum, "MIMO capacity with interference," IEEE $J$. Select. Areas Commun., vol. 21, no. 5, pp. 793-801, June 2003.

[7] H. Zhang and H. Dai, "Cochannel interference mitigation and cooperative processing in downlink multicell multiuser MIMO networks," EURASIP Journal on Wireless Communications and Networking, no. 2, pp. 222-235, 2004.

[8] G. Foschini, H. Huang, K. Karakayali, R. Valenzuela, and $\mathrm{S}$. Venkatesan, "The value of coherent base station coordination," in Conference on Information Sciences and Systems, The John Hopkins University, USA, 16-18 Mar 2005.

[9] M. Karakayali, R. A. Valenzuela, and G. J. Foschini, "Network co-ordination for spectrally efficient communication in cellular systems," IEEE Wireless Commun. Mag., vol. 13, no. 4, pp. 56-61, Aug. 2006.

[10] H. Huang, A. Hottinen, M. Shafi, P. J. Smith, M. Trivellato, and R. Valenzuela, "System aspects and performance of MIMO in cellular networks," submitted to IEEE Trans. Wireless Comm., 2008.

[11] M. Costa, "Writing on dirty paper," IEEE Trans. Inform. Theory, vol. 29, no. 3, pp. 439-441, May 1983.

[12] R. Yates, "A framework for uplink power control in cellular radio systems," IEEE J. Select. Areas Commun., vol. 13, no. 7, pp. 1341-1347, Sept. 1995.

[13] S. Ulukus and R. Yates, "Stochastic power control for cellular radio systems," IEEE Trans. Commun., vol. 46, no. 6, pp. 784 798, June 1998

[14] A. Gjendemsjo, D. Gesbert, G. E. Oien, and S. G. Kiani, "Optimal power allocation and scheduling for two-cell capacity maximization," in Proc. 4th International Symposium on Modeling and Optimization in Mobile, Ad Hoc and Wireless Networks, Boston, USA, Apr. 2006, p. 16.

[15] A. Gjendemsjo, D. Gesbert, and G. E. Oien, "Binary power control for multi-cell capacity maximization," in Proc. Signal Processing Advances in Wireless Communications, Helsinki, Finland, 17-20 June 2007, pp. 1-5.

[16] M. Ebrahimi, M. A. Maddah-Ali, and A. K. Khandani, "Power allocation and asymptotic achievable sum-rates in single-hop wireless networks," in Proc. 40th Annual Conference on Information Sciences and Systems, Princeton, NJ, USA, Mar. 2006, p. 498503 .

[17] M. Chiani, M. Z. Win, and H. Shin, "Capacity of MIMO systems in the presence of interference," in Proc. IEEE Global Telecommunications Conf., San Francisco, CA, USA, Nov 27 - Dec 1, 2006, pp. 1-6.

[18] N. Badruddin, J. Evans, and S. Hanly, "Maximizing sum rate for two interfering wireless links," in Australian Communications Theory Workshop, Christchurch, NZ, 30 Jan-1 Feb 2008, pp. $75-81$.

[19] M. Chiani, M. Z. Win, and H. Shin, "MIMO networks: the effects of interference," submitted to IEEE Trans. Inform. Theory, 2008

[20] S. Boyd and L. Vandenberghe, Convex Optimization, 1st ed. Cambridge, UK: Cambridge University Press, 2004.

[21] K. B. Petersen and M. S. Pedersen, The Matrix Cookbook, 2008. [Online]. Available: http://matrixcookbook.com

[22] P. J. Smith, T. W. King, and L. M. Garth, "Capacity and fairness of MIMO broadcast algorithms in shadow fading environments," in Proc. IEEE Global Telecommunications Conf., Washington DC, USA, 26-30 Nov, 2007, pp. 3616-3622. 